\title{
Enhanced therapeutic effect of an antiangiogenesis peptide on lung cancer in vivo combined with salmonella VNP20009 carrying a Sox2 shRNA construct
}

\author{
Changhong Zhao ${ }^{1}$, Junjin $\mathrm{He}^{1}$, Haoran Cheng ${ }^{1}$, Zhaohao Zhu ${ }^{1}$ and Hanmei $\mathrm{Xu}^{1,2^{*}}$
}

\begin{abstract}
Background: HM-3 is a polypeptide inhibiting angiogenesis. Recent reports suggest that the antitumor effect of angiogenesis inhibitors administered alone might be limited. Cancer stem cells can survive the lack of oxygen and nutrients. To achieve better anti-tumor effect, HM-3 was administered in combination with the attenuated Salmonella typhimurium VNP20009 transformed with a shRNA construct against sex determining region Y-box 2 (Sox2).

Methods: Cell invasion assay and soft agar colony formation assay were used to assess the migration and growth capability of A549 cells once Sox2 was knocked down with the shRNA construct. The shRNA construct targeting Sox2 was transformed into VNP20009. After the mouse xenograft model of A549 was established, HM-3 was co-administered with VNP20009 carrying the shRNA construct. The growth of tumor was checked to compare the effectiveness of different therapies. Western blotting assay and immunohistochemistry staining of the tumor tissue were used to measure the levels of proteins associated with the apoptosis pathway.

Results: Sox2 was necessary for the migration and growth of A549 cells. The expression of Sox2 was down regulated in the tumor tissue of the combined treatment group of HM-3 with VNP20009 carrying the Sox2 shRNA construct. Together with the accumulation of salmonella in tumor and the inhibition of angiogenesis by HM-3, more tumor cells went through cell apoptosis with increased expression of Bax, cleaved Caspase 3 and decreased expression of $\mathrm{BCl} 2$.

Conclusions: The results suggest the combination of antiangiogenesis agent $\mathrm{HM}-3$ with gene therapy targeting Sox2 delivered by salmonella as a promising strategy for the treatment of lung cancer.
\end{abstract}

Keywords: Sox2, Lung cancer, Antiangiogenesis, Salmonella typhimurium, Apoptosis

\section{Background}

Lung cancer is one of the leading causes of cancerrelated death throughout the world. The average 5-year survival rate of non-small cell lung carcinoma (NSCLC) which accounts for $85 \%$ of lung cancers is only $4 \%$, highlighting the need for more effective treatment

\footnotetext{
* Correspondence: 13913925346@126.com

${ }^{1}$ The Engineering Research Center of Peptide Drug Discovery and Development, China Pharmaceutical University, 24 Tongjia Xiang, Nanjing 210009, People's Republic of China

${ }^{2}$ State Key Laboratory of Natural Medicines, Ministry of Education, China Pharmaceutical University, 24 Tongjia Xiang, Nanjing 210009, People's Republic of China
}

options [1]. HM-3 is an 18-amino acid peptide generated by the fusion of the Arg-Gly-Asp (RGD) sequence to the $\mathrm{C}$-terminus of an endostatin-derived peptide. Our previous research has shown that HM-3 has no effect on the growth of tumor cells in vitro. However, HM-3 can inhibit tumor growth by inhibiting angiogenesis in vivo $[2,3]$. The binding of $\mathrm{HM}-3$ to integrin $\alpha \mathrm{v} \beta 3$ expressed on the surface of vascular endothelium cells (VECs) results in the down-regulation of MEK1 and AKT1, leading to the inhibition of the migration of VECs [4-6]. HM-3 has been approved for phase I clinical study in China (Register number: CTR20150368). Recent studies 
reported that the antitumor effect of angiogenesis inhibitors (AIs) administered alone might be limited implicating that additional tumor targeting drugs should be coadministered with AIs [7-9].

Tumor growth is sustained by a small number of tumor-initiating cells known as cancer stem cells (CSCs). Sox2, a gene expressed at high level in stem cells, also plays a vital role in the regenesis of lung CSCs [10-12]. It has been reported that the growth and metastasis of certain cancer cells were suppressed when Sox 2 was down regulated suggesting Sox 2 may be a potential target for cancer treatment $[13,14]$. Finding an effective way to deliver Sox 2 shRNA constructs into lung cancer cells may achieve better anti-tumor effect. Among the gene therapy delivery systems reported, attenuated salmonella offers more advantages as it can preferentially accumulate and amplify in solid tumor with little toxicity to normal organs. Also, it has been used as gene therapy delivering system for many shRNA constructs targeting different genes such as indoleamine 2, 3-dioxygenase 1 (IDO1) [15], multiple drug resistance 1 (MDR1) [16] and murine double minute 2 (MDM2) [17].

Here, we explored the antitumor effect of the novel combination therapy of HM-3 with VNP20009 carrying a Sox2 shRNA construct in a mouse xenograft model of NSCLC. Our results showed that the combined therapy had the optimal antitumor effect compared to HM-3 alone or docetaxel alone. The optimal therapeutic effect was achieved through accumulation of VNP20009 in tumor tissue, suppression of the expression of Sox 2 and inhibition of angiogenesis in tumors. More tumor cells went through cell apoptosis in the combined treatment group. Also, no apparent toxic side-effect was observed in the mice.

\section{Methods}

\section{Cell lines, animals and drugs}

The NSCLC cell line A549 was purchased from Shanghai Institute of Cell Biology, Chinese Academy of Sciences (Shanghai, China). A549 cells were maintained in RPMI1640 medium containing $10 \%$ FBS. Athymic BALB/c nude female mice (5 weeks) were purchased from CAVENS (Changzhou, China). HM-3 (sequence: IVRRADRAAVPGGGGRGD) with purity of more than $99 \%$ was chemically synthesized in our lab. The antibodies against Sox2 and CD31 were purchased from Santa Cruz (Dallas, USA). The antibodies against $\mathrm{Bax}$ and $\mathrm{Bcl} 2$ were obtained from Wanleibio (Dalian, China). The antibody against cleaved Caspase 3 was obtained from Elabscience (Wuhan, China). Docetaxel Injection was purchased from Hengrui (Jiangsu, China).

\section{Plasmids and bacterial strains}

The mammalian shRNA expression plasmid (pGPU6/ Neo) targeting Sox2 was constructed by GenePharma
(Shanghai, China). The shRNA sense sequence targeting Sox2 was TGGACAGTTACGCGCACATGA as reported and the scrambled shRNA sense sequence was GTTCTCCGAACGTGTCACGT [18]. The scrambled shRNA construct was referred as shScr while the Sox2 shRNA construct was referred as shSox 2 . The shRNA construct was electroporated into VNP20009 with a Gene Pulser Xcell system (Bio-Rad) at $2.5 \mathrm{kV}, 186 \mathrm{ohms}$ [15]. VNP20009 transformed with the shScr (shScr-V) and VNP20009 transformed with the shSox2 (shSox2-V) was cultured in Luria-Bertani (LB) broth supplemented with ampicillin $(50 \mu \mathrm{g} / \mathrm{ml})$ [19]. The shRNA constructs were isolated and the targeting sequence was confirmed by sequencing. To calculate the number of colony forming unit (cfu) in culture, an optical density of 1.0 measured at $600 \mathrm{~nm}$ equal to $10^{9} \mathrm{cfu} / \mathrm{ml}$ was used.

\section{Cell invasion assay and soft agar colony formation assay}

The cell invasion assay was performed using 24-transwell chambers (Corning, Newyork, USA). The procedures were carried out as previously reported [12]. For soft agar colony formation assay, a layer of media with $0.5 \%$ agarose was first plated onto the bottom of a 6 -well plate. $1 \times 10^{4}$ cells/well were plated in a top layer of media with $0.33 \%$ agarose. After stained with $0.005 \%$ crystal violet, images were taken under an inverted microscope (Olympus IX53) after three weeks. The number of colonies was counted from multiple random fields.

\section{Tumor models and treatment}

$2 \times 10^{6}$ A549 cells were injected subcutaneously (s.c) on the right flanks of the nude mice. The width (W) and length (L) of each tumor was measured with a vernier caliper. Tumor volume (TV) was determined with the formula: $\mathrm{TV}=\mathrm{L} \times \mathrm{W}^{2} / 2$. When the mean tumor volumes reached $300 \mathrm{~mm}^{3}$, the mice were randomly assigned into seven groups ( $n=6$ at least): (1) the mice received $200 \mu \mathrm{l}$ normal saline (NS) intravenously as the normal control; (2) $2.5 \times 10^{6} \mathrm{cfu}$ of shScr-V diluted in NS was administered intravenously on day 1 ; (3) $2.5 \times$ $10^{6} \mathrm{cfu}$ of shSox2-V diluted in NS was administered intravenously on day 1 ; (4) HM-3 diluted in NS was administered intravenously at a dose of $3 \mathrm{mg} / \mathrm{kg} /$ day; (5) both HM-3 and shScr-V was administered as for their individual treatment regimens; (6) both $\mathrm{HM}-3$ and shSox2-V was administered as for their individual treatment regimens; (7) docetaxel was administered intravenously at a dose of $10 \mathrm{mg} / \mathrm{kg}$ every four days for three injections. All the groups were listed in Table 1. Inhibition rate $=[$ (tumor weight of control group - tumor weight of experimental group)/tumor weight of control group] $\times 100 \%$. 
Table 1 Treatment groups

\begin{tabular}{ll}
\hline Group & Treatment \\
\hline Mock & Normal saline (NS) was administered as control \\
shScr-V & $\begin{array}{l}\text { VNP20009 carrying the scrambled shRNA construct } \\
\text { was administered } \\
\text { ShSox2-V }\end{array}$ \\
VNP20009 carrying the Sox2 shRNA construct \\
was administered \\
HM-3 & HM-3 was administered \\
HM-3 + shScr-V & Both HM-3 and shScr-V were administered \\
Do-3 + shSox2-V & Both HM-3 and shSox2-V were administered \\
\hline
\end{tabular}

\section{Assay of VNP20009 in tissues and blood}

After the A549 tumor model in nude mice was established, VNP20009 was administered by intravenous injection at a dose of $2.5 \times 10^{6} \mathrm{cfu}$ per mouse. On days 2, 7 and 14 after injection, tumors and major organs were aseptically removed, weighted and homogenized in PBS. $1 \mathrm{ml}$ homogenized tissue of the same weight (100 mg) was diluted in gradient and $100 \mu \mathrm{l}$ was plated onto LB agar plates containing ampicillin (50 $\mu \mathrm{g} / \mathrm{ml})$. In the case of blood analysis from mice, 200 $\mu \mathrm{l}$ blood was collected as the mice were killed and was plated directly onto the LB plates. The LB plates were incubated at 37 Celsius overnight and the bacteria colonies were quantified.

\section{Western blotting assay (WB)}

The tumor samples were snapped into small pieces and incubated with RIPA cell lysis buffer supplemented with protease inhibitors on ice for $20 \mathrm{~min}$. After that, the samples were homogenized with a glass tissue grinder. After centrifuging $(14,000 \times \mathrm{g})$ for $20 \mathrm{~min}$, the supernatant was removed and boiled with protein loading buffer. The western blotting was carried out as described before [5]. ImageJ software (Wayne Rasband, USA) was used to analyze the result. The relative protein expression was calculated by dividing the optical density of the protein analysed with the optical density of $\beta$-actin of the same group. The results of three independent experiments were calculated.

\section{Immunohistochemistry (IHC)}

Tumor and organ tissue was fixed in $4 \%$ formalin and embedded in paraffin. Sections were deparaffinized and rehydrated. For hematoxylin and eosin (H\&E) staining, sections were stained in hematoxylin and then counterstained with eosin. For immunohistochemistry staining, after treatment with $3 \% \mathrm{H}_{2} \mathrm{O}_{2}$, sections were boiled in $10 \mathrm{mM}$ sodium citrate buffer $(\mathrm{pH}$ 6.0) for antigen retrieval. After blocking, sections were incubated with the primary antibody as recommended by the supplier, washed with PBS, and then incubated with the horseradish peroxidase labeled secondary antibody. Sections were finally incubated with $\mathrm{DAB}$ and counterstained with hematoxylin.

\section{Statistical analysis}

All the data was collected and processed with the Statistical Program for Social Sciences (SPSS version 13.0, Chicago, USA). The difference between samples of the same group was tested by one-way analysis of variance. It would be considered statistically significant when $P<0.05$ and statistically highly significant when $P<0.01$.

\section{Results}

The invasion and anchorage-independent growth capability of A549 cells was inhibited as Sox 2 was knocked down

To confirm the effectiveness of the shRNA constructs, the expression of Sox 2 at protein level in A549 cells was analysed with WB after transfected with the shRNA constructs. Compared to the A549 cells transfected with shScr, the expression of Sox 2 was reduced by more than $80 \%$ in the cells transfected with shSox2 (Fig. 1a, $P<$ 0.01 ). The number of cells that migrated was $236.33 \pm$ 26.08 for A549 cells transfected with shScr, while it was $45.23 \pm 12.50$ for A549 cells transfected with shSox2 (Fig. 1b, $P<0.01$ ). The number of colonies that formed was $225.33 \pm 62.98$ for A549 cells transfected with shScr, while it was $57.33 \pm 12.01$ for cells transfected with shSox2 (Fig. 1c, $P<0.05$ ). Sox2 plays an important role in regulating the migration and anchorage-independent growth of A549 cells. Therefore, Sox 2 may be considered as a potential target for the treatment of lung cancer.

\section{VNP20009 selectively accumulated in tumors}

To ensure that VNP20009 transformed with the shRNA expression plasmid still preferentially accumulated in solid tumor tissues, the distribution of shScr$\mathrm{V}$ in the A549 xenografts and major organs of mice treated with shScr-V was monitored. On days 2 postinjection, the amount of shScr-V in tumors was significantly higher than it was in spleen and other organs (Fig. 2a). No shScr-V was detected in the blood on days 2 suggesting that the environment in blood was not well adapted by VNP20009 [20]. Quantitative analyses showed that on days 2, 7 and 14 after injection, shScr-V could maintain its accumulation in tumors over spleen and other organs at a ratio greater than 1000:1 (Fig. 2b, $P<0.01$ ).

\section{Antitumor activity of HM-3 combined with shSox2-V in vivo}

After the mouse xenograft model of A549 was established, the mice were assigned randomly to seven groups 


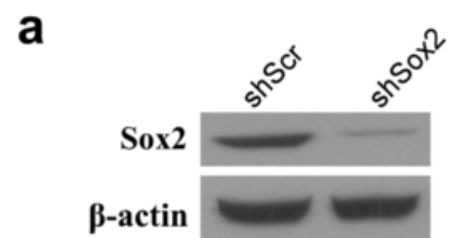

b

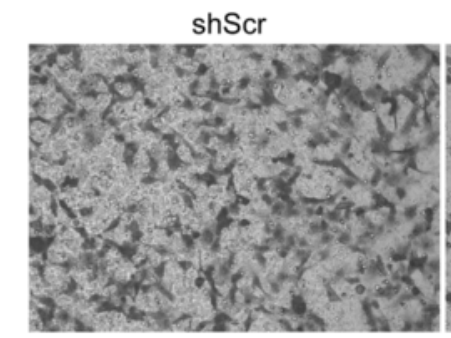

c

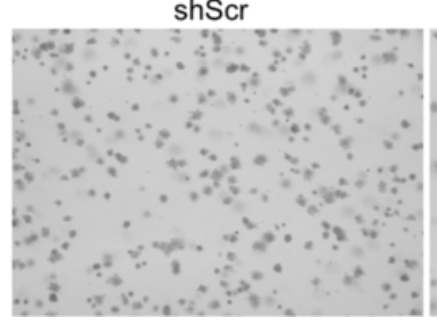

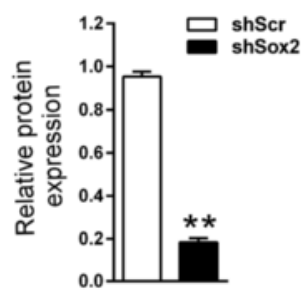

shSox2
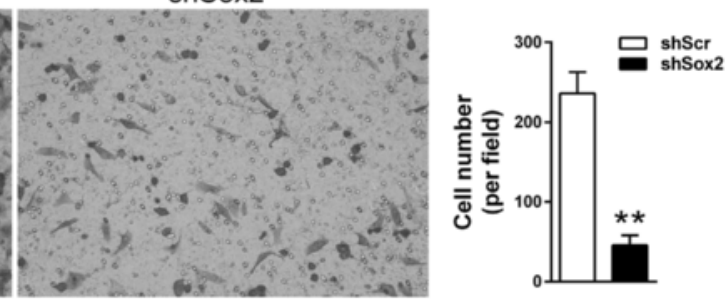

shSox2
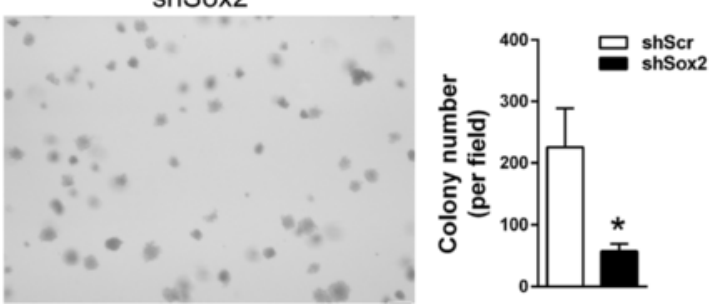

Fig. 1 The migration and proliferation potential of A549 cells was inhibited as Sox2 was down regulated. a The expression of Sox2 at protein level in A549 cells was analysed using western blot after transfection. shScr, A549 cells transfected with shScr. shSox2, A549 cells transfected with shSox2. The quantification assay of WB results was shown as mean \pm SD obtained from three repeated experiments. ${ }^{* *} P<0.01$ vs shScr group. $\mathbf{b}$ The migration capability of A549 cells was reduced after transfected with shSox2. Original magnification $\times 200$. The quantification data shown was mean \pm SD obtained from three fields. ${ }^{*} P<0.01$ vs shScr group. $\mathbf{c}$ The colony forming capability of A549 cells was inhibited after transfected with shSox2. Original magnification $\times 200$. The quantification data shown was mean \pm SD obtained from three fields. $* P<0.05$ vs shScr group

(Fig. 3a and Table 1). HM-3 was administered alone and in combination with shScr-V or shSox2-V. Although the tumor growth of the shSox2-V, HM-3 + shScr-V and docetaxel groups all had been suppressed compared to the mock group $(P<0.05$, respectively), the best therapeutic effect was obtained in the HM-3 + shSox $2-\mathrm{V}$ group (Fig. 3b, $P<0.01$ ). The mean tumor volume of the HM$3+$ shSox $2-\mathrm{V}$ group was $682.37 \mathrm{~mm}^{3}$, while that in the mock, shScr-V, shSox2-V, HM-3, HM-3 + shScr-V and docetaxel groups was $1516.32 \mathrm{~mm}^{3}, 1194.84 \mathrm{~mm}^{3}$, $1043.85 \mathrm{~mm}^{3}, 1129.68 \mathrm{~mm}^{3}, 906.14 \mathrm{~mm}^{3}$ and 822.78 $\mathrm{mm}^{3}$ respectively at the end of the treatment (Fig. 3c). The difference of tumor volume between the HM-3 + shScr-V and HM-3 + shSox2-V group was statistically significant $(P<0.05)$ implicating that the shSox 2 carried by shSox $2-\mathrm{V}$ contributing to the inhibition of tumor growth. The mean weight of tumors of the HM-3 + shSox2-V group was $0.586 \mathrm{~g}$, while that in the mock, shScr-V, shSox2-V, HM-3, HM-3 + shScr-V and docetaxel groups was $1.420 \mathrm{~g}, 1.075 \mathrm{~g}, 0.899 \mathrm{~g}, 1.037 \mathrm{~g}$, $0.783 \mathrm{~g}$ and $0.737 \mathrm{~g}$ respectively (Fig. 3d). Both shScr-V and shSox $2-\mathrm{V}$ could enhance the antitumor effect of
HM-3 as compared to the mock group $(P<0.05$ and $P<$ 0.01 , respectively). The best antitumor effect was achieved in the HM-3 + shSox $2-\mathrm{V}$ group. Due to the variance of tumor weight in mice, the difference of tumor weight between the HM-3 + shScr-V and HM-3 + shSox $2-\mathrm{V}$ group was not statistically significant.

\section{The expression of Sox 2 and microvessel formation in the A549 xenografts of different treatment groups}

The expression of Sox 2 in the tumor tissues was examined by WB and IHC. In contrast to the groups treated with NS, HM-3 and shScr-V, Sox 2 expression was much lower in the HM-3 + shScr-V, HM-3 + shSox2-V and docetaxel groups (Fig. 4a, $P<0.05$ ). When coadministered with HM-3, shSox2-V could more effectively inhibit the expression of Sox 2 compared to shScr-V $(P<0.01)$. The results indicated that the successful transfection of Sox 2 shRNA by VNP20009 in vivo. Docetaxel is a microtubule inhibitor that inhibits the disassociation of microtubule during cell division. The down regulation of Sox2 in the docetaxel group might result from the cell cycle arrest and cell death induced by 


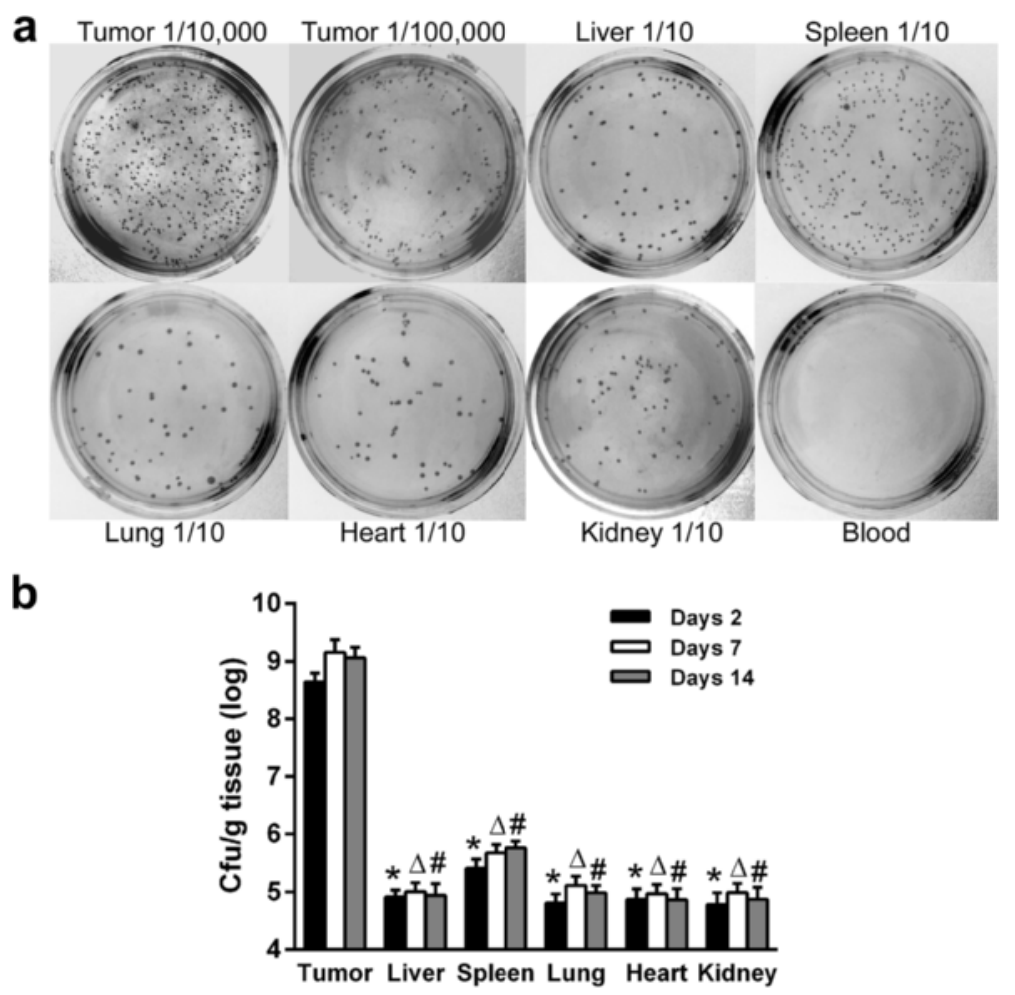

Fig. 2 VNP20009 preferably accumulated in tumors in vivo. a The representative images of LB agar plates planted with different homogenized tissue after serial dilution or blood on days 2 after injection. The dilution factor was marked, 1/10 for ten fold dilution. $\mathbf{b}$ The quantitative analyses of the bacterium count by cfu/g tissue were presented for days 2,7 and 14 post injections. Data shown was mean \pm SD obtained from three independent experiments. $* P<0.01$ vs the tumor group on days $2, \Delta P<0.01$ vs the tumor group on days $7, \# P<0.01$ vs the tumor group on days 14

docetaxel rather than a direct effect. In consistent with the WB result, the number of Sox2 positive cells was also reduced in the HM-3 + shScr-V, HM-3 + shSox2-V and docetaxel groups (Fig. 4b). The number of Sox2 positive cells per field for the mock, shScr-V, shSox2-V, HM-3, HM-3 + shScr-V, HM-3 + shSox2-V and docetaxel groups was 39.5, 39.25, 30.75, 41.75, 22.25, 10.5 and 13.25 respectively (Fig. 5a).

CD31 has been used extensively to evaluate the degree of angiogenesis in tumors. As shown in the WB and IHC results, the amount of CD31 was much lower in the HM-3, HM-3 + shScr-V and HM-3 + shSox2-V groups compared to those treated with NS, shScr-V, shSox2-V and docetaxel (Fig. $4 \mathrm{a}$ and c, $P<$ 0.05). The microvessel number per field for the mock, shScr-V, shSox2-V, HM-3, HM-3 + shScr-V, HM-3 + shSox2-V and docetaxel groups was 10.25, $9.75, \quad 10.75, \quad 3.5, \quad 3.25, \quad 2.5$ and 9.75 respectively (Fig. 5b). The results confirmed that HM-3 could inhibit the microvessel formation in tumors. The difference of microvessel density between HM-3 + shScr-V and HM-3 + shSox2-V group was not significant suggesting that the antiangiogenesis activity of HM-3 was not affected in both groups.
Effects of HM-3 with shSox2-V on the expression of apoptosis-related protein in the A549 xenografts

Histological examination showed large areas of necrosis in the HM-3 + shScr-V, HM-3 + shSox2-V and docetaxel groups (Fig. 6a). The accumulation of salmonella and co-expression of Sox 2 shRNA enhanced the therapeutic effect of HM-3 on the growth of tumor. It has been reported that the silencing of Sox2 leading to the up regulation of apoptotic marker cleaved Caspase 3 (C-caspase 3 ) in lung cancer [21]. Also, it has been found that VNP20009 could migrate away from vasculature and induce apoptosis in tumor tissue [22]. Together with HM3 inhibiting the microvessel formation in tumors, we assumed that tumor cells were more susceptible to the induction of cell death. The expression of apoptosisrelated protein $\mathrm{C}$-caspase $3, \mathrm{Bax}$ and $\mathrm{Bcl} 2$ was examined with WB (Fig. 6b). Compared to the mock group, the expression of apoptosis stimulators Bax and C-caspase 3 was much higher in the HM-3 + shSox2-V and docetaxel groups $(P<0.05)$. In contrast, the expression of apoptosis inhibitor $\mathrm{Bcl} 2$ was much lower in the HM-3 + shSox2-V and docetaxel groups $(P<0.05)$. Those results suggested that the apoptosis pathway was activated in the HM-3 + shSox $2-\mathrm{V}$ and docetaxel groups. The 


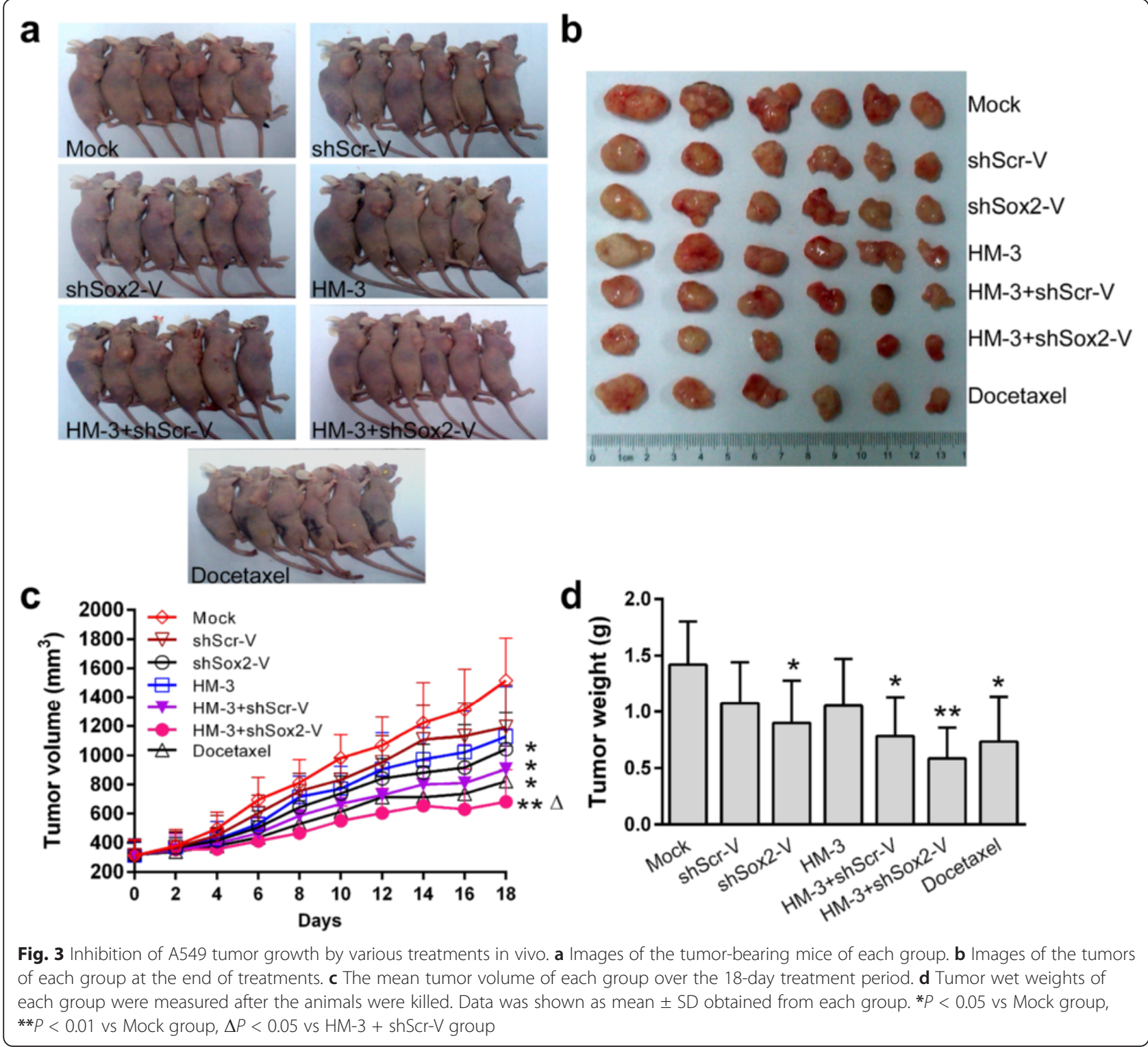

difference of the expression of Bax and C-caspase 3 between the HM-3 + shScr-V and HM-3 + shSox2-V group was statistically highly significant $(P<0.01$, respectively) indicating that shSox 2 contributed to the activation of the apoptosis pathway.

\section{Systematic toxicity in the mice treated with $\mathrm{HM}-3+$ shSox2-V}

Previous study has found that VNP20009 administered alone can be tolerated by athymic mice [23]. The systematic safety when HM-3 and shSox2-V administered together still requires further verification. We compared the body weight of mice in the HM-3 + shSox $2-\mathrm{V}$ group to the other groups (Fig. 7a). Those mice treated with docetaxel had the lowest mean body weight at the end of the study. The body weight of the mice treated with
HM-3 was about the same as the mock group. Although the mean body weight of mice in the HM- $3+$ shSox $2-\mathrm{V}$ group was lower than the mock group, the difference was not statistically significant. After the tumor-bearing mice were sacrificed, their hearts, livers, spleens, kidneys and lungs were harvested and examined by H\&E staining (Fig. 7b). As shown in the result, the combined treatment with HM-3 and shSox2-V had little toxicity to the major organs compared to the mock group.

\section{Discussion}

At the early stages of tumor progression, microvessels are formed around tumor. If angiogenesis inhibitors are administered at this stage, the growth of tumor could be effectively inhibited. However, the microenvironment in the inner tumor mass changes as tumor progresses. The 


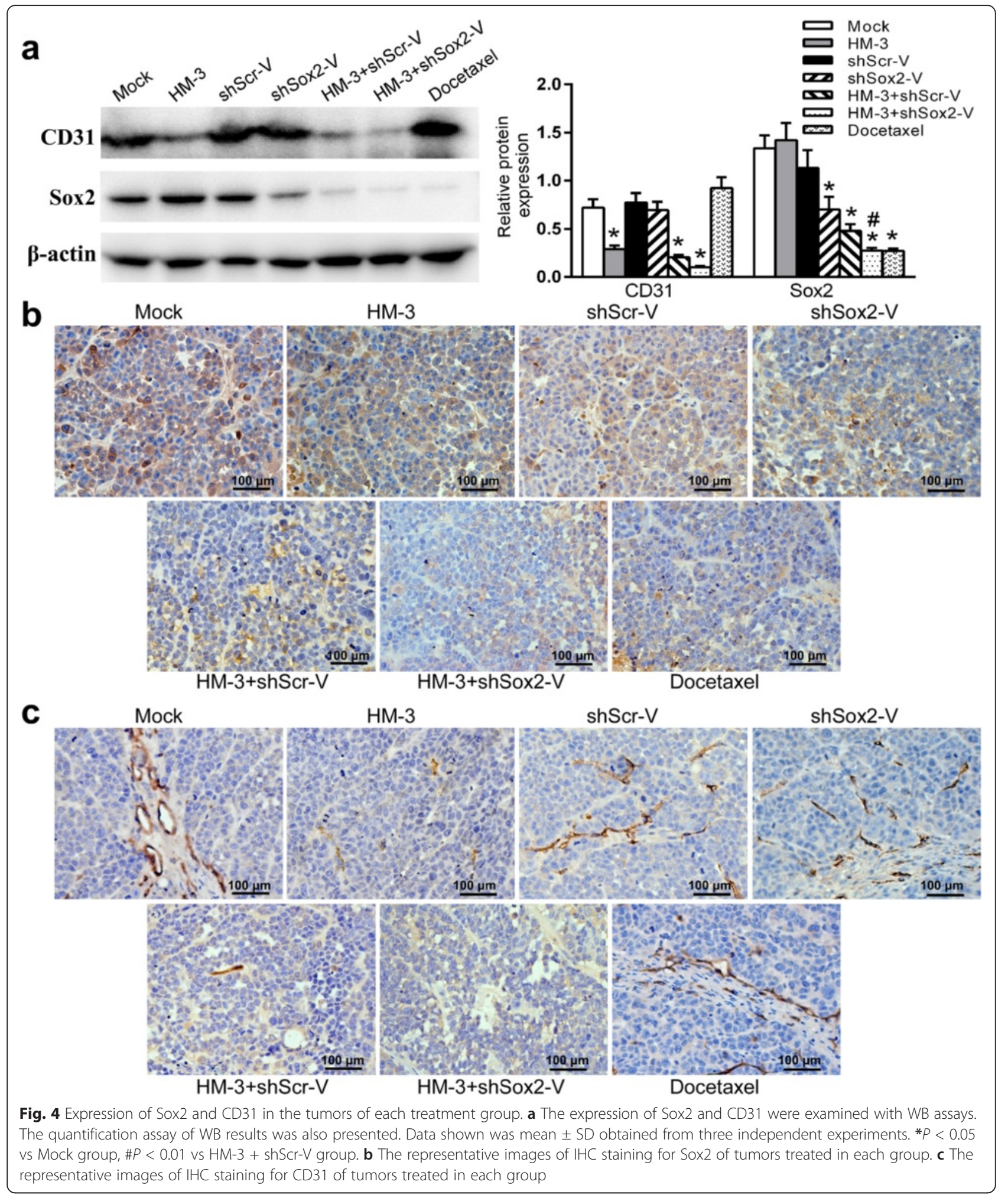

actions of antitumor drugs may be affected as the expression of various factors and microRNAs are influenced by the microenvironment [24, 25]. Also, the survival of CSCs further limits the effectiveness of chemotherapy and anti-angiogenesis therapy [26-28]. In this study, the treatment was started after the tumor volume was greater than $300 \mathrm{~mm}^{3}$. On one hand, polypeptide HM-3 was administered to suppress the microvessel 


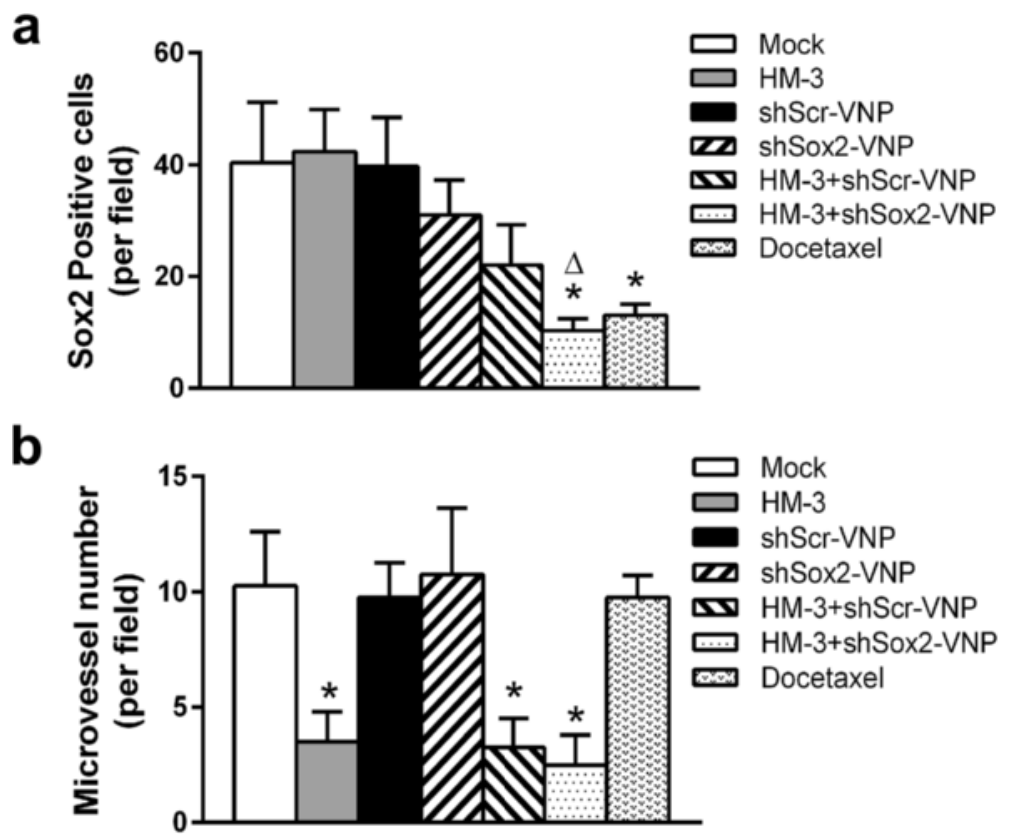

Fig. 5 The quantification analysis of the IHC result. a The Sox2 positive cells per field were counted. The data shown was mean \pm SD obtained from four fields. ${ }^{*} P<0.05$ vs Mock group, $\Delta P=0.055$ vs HM-3 + shScr-V group. $\mathbf{b}$ The microvessel number per field was counted. The data shown was mean $\pm \mathrm{SD}$ obtained from four fields. ${ }^{*} P<0.05$ vs Mock group

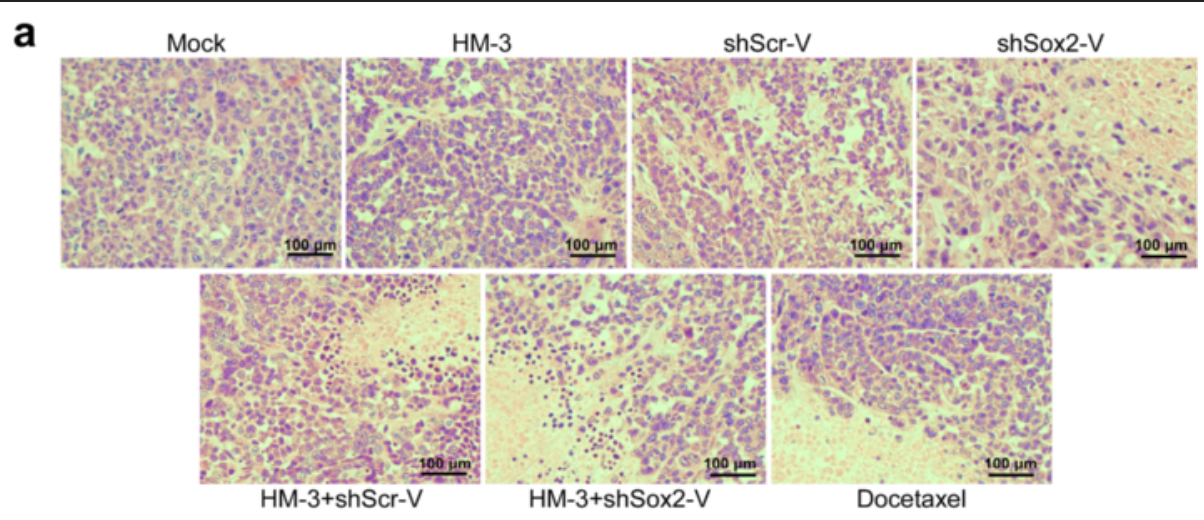

b

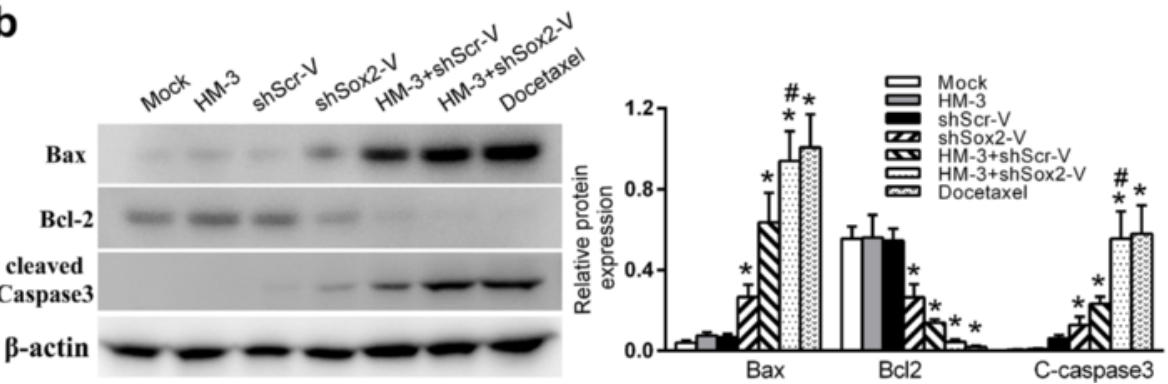

Fig. 6 The induction of cell apoptosis in tumors. a Representative images of H\&E staining of tumors of each treatment group. $\mathbf{b}$ The expression of Bax, Bcl2 and cleaved Caspase 3 (C-caspase 3) were examined with WB assays. The quantification assay of WB results was also presented. The data shown was mean \pm SD obtained from three independent experiments. ${ }^{*} P<0.05$ vs Mock group, $\# P<0.01$ vs HM-3 + shScr-V group 
a

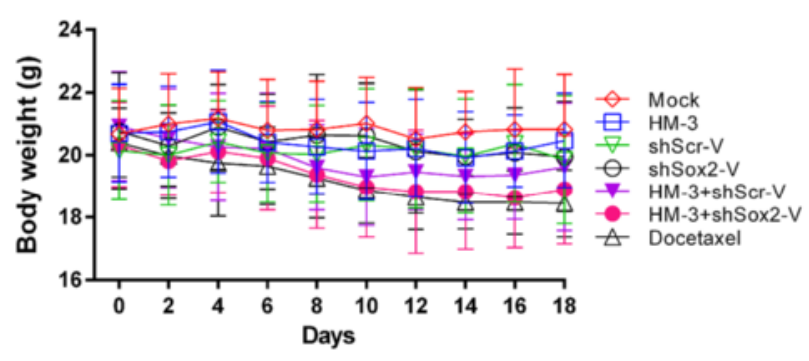

b

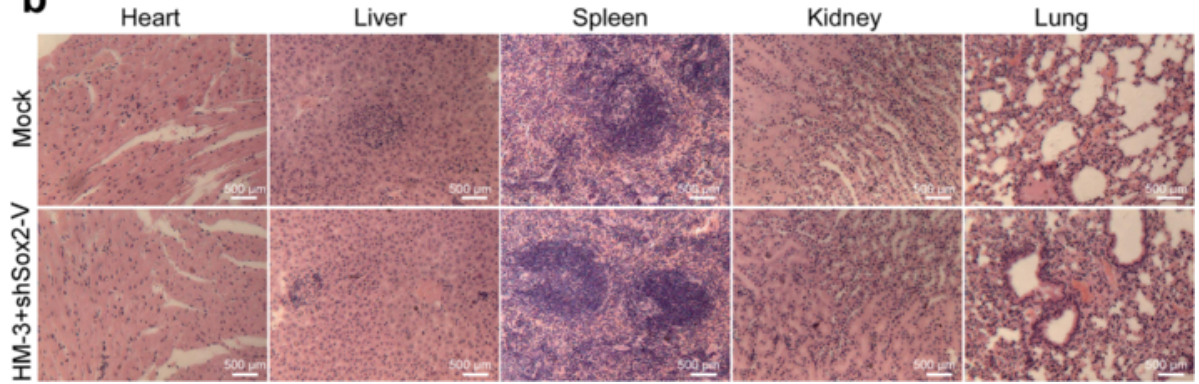

Fig. 7 Systematic toxicity in the mice of each treatment group. a The mean body weight of tumor-bearing mice treated in each group was measured during the study. The data shown was mean \pm SD obtained from each group. $\mathbf{b}$ The images of H\&E staining of major organs of mice treated with NS and $\mathrm{HM}-3+$ shSOX2-V

formation in tumor which cut the supply of oxygen and nutrients for tumor growth. On the other hand, attenuated facultative anaerobe VNP20009 was administered which can specifically accumulate in tumor because of its natural tropism for hypoxic areas in contrast to chemo drugs [29]. The inhibition of microvessel formation in tumor could not affect the tumor targeting potential of VNP20009 as it was cleared away from the blood quickly and accumulated in tumors shortly after injection. To further inhibit the survival of CSCs in tumor tissue, a shRNA construct targeting Sox2 was transformed into VNP20009. The expression of Sox2 was down regulated in the tumor tissue of the combined treatment group while with much smaller tumor volume.

The engineered salmonella enhanced the antitumor effect of HM-3 on lung cancer compared to the individual therapy group. The inhibition rate of the HM-3 + shSox2-V group was $58.73 \%$, while that in the shScr-V, shSox2-V, HM-3, HM-3 + shScr-V and docetaxel groups was $24.3 \%, 36.69 \%, 26.97 \%, 44.86 \%$ and $48.1 \%$ respectively. Our results suggested that this combination was effective in inhibiting tumor growth in vivo. According to our knowledge, this was the first report that attenuated salmonella with a shRNA construct targeting Sox2 was co-administered with a polypeptide inhibiting angiogenesis in the treatment of lung cancer.

Sox2 is a poor prognostic indicator in stage I lung cancer [30]. Also, it has been reported that the expression of Sox2 promoted drug resistance of certain tumors [31, 32]. By knocking down the expression of Sox 2 with shRNA, the growth of lung cancer cells was inhibited. In consistent with previous reports, the down regulation of Sox2 was related to the expression of apoptosis related marker $\mathrm{C}$-caspase 3 [21]. However, our results suggested that knocking down Sox 2 alone was not enough to significantly inhibit A549 tumor growth in vivo. Considering the redundancy of signaling factors in tumor cells, other members of the same protein family or different pathways may compensate for the loss of Sox 2 leading to the survival of tumor cells [33, 34].

Cell death mediated by apoptosis is important in the regulation of tumor growth. It has been reported that VNP20009 could migrate from the vascular and induce apoptosis in tumor [22]. Our results showed that the apoptosis induced by VNP20009 alone was not evident. This might be due to the difference of tumor model and treatment regimens. More tumor cell went through apoptosis in the HM-3 + shSox $2-\mathrm{V}$ group implicating that the inhibition of angiogenesis by HM-3 had synergistic antitumor effects with shSox2-V. The cell apoptosis pathway was also activated in the group treated with docetaxel due to its cytotoxicity. It has been reported that the combination therapy of cytotoxic agents with salmonella was effective in inhibiting the growth of prostate cancer [17]. However, whether this combination can be applied to lung cancer still requires further verification.

\section{Conclusions}

Our study suggested that HM-3 together with a gene therapy targeting Sox2 delivered by VNP20009 was an 
attractive treatment option for patients with lung cancer. However, to achieve better antitumor effect, multitargeting therapies with cytotoxic drugs and the use of other gene therapy delivering system may be considered [35]. Also, the key factors of other signal pathways that are involved in lung cancer growth can be evaluated as potential targets [36].

\begin{abstract}
Abbreviations
Als, angiogenesis inhibitors; C-caspase 3, cleaved Caspase 3; Cfu, colony forming unit; CSCs, cancer stem cells; H\&E, hematoxylin and eosin; IDO1, indoleamine 2. 3-dioxygenase 1; IHC, immunohistochemistry; L, length; LB, Luria-Bertani; MDM2, murine double minute 2; MDR1, multiple drug resistance 1; NS, normal saline; NSCLC, non-small cell lung carcinoma; RGD, Arg-Gly-Asp; s.C, subcutaneously; shScr, the scrambled shRNA construct; shScr-V, VNP20009 transformed with the shScr; shSox2, the Sox2 shRNA construct; shSox2-V, VNP20009 transformed with the shSox2; Sox2, sex determining region Y-box 2; TV, tumor volume; VECs, vascular endothelium cells; W, width; WB, western blotting assay
\end{abstract}

\section{Acknowledgements}

This work was mainly supported by 863 High-Technology Development Planning (No. SQ2011SF11B02030), the Project Program of State Key Laboratory of Natural Medicines (No. SKLNMBZ201403) and the National Science and Technology Major Projects of New Drugs (No. 2012ZX09103301004 and No. 2014ZX09508007) in China. This project was also funded by the Priority Academic Program Development of Jiangsu Higher Education Institutions (PAPD).

\section{Authors' contributions}

$\mathrm{HMX}$ and $\mathrm{CHZ}$ conceived and designed the experiments. $\mathrm{CHZ}$, JJH and HRC carried out the in vivo study. $\mathrm{CHZ}$ and $\mathrm{ZHZ}$ performed the $\mathrm{WB}, \mathrm{IHC}$ and statistical analysis. $\mathrm{CHZ}$ and $\mathrm{HMX}$ prepared the manuscript. All authors read and approved the manuscript.

\section{Competing interests}

The authors declare that there are no competing interests.

\section{Ethics approval and consent to participate}

The study involved with animals was approved by the Animal Ethics Committees of China Pharmaceutical University. All the experimental animals were kept according to the Guide for the Care and Use of Laboratory animals.

Received: 29 March 2016 Accepted: 21 June 2016

Published online: 02 July 2016

\section{References}

1. Harvey RD. Immunologic and clinical effects of targeting PD-1 in lung cancer. Clin Pharmacol Ther. 2014;96:214-23.

2. Xu HM, Yin R, Chen L, Siraj S, Huang X, Wang M, et al. An RGD-modified endostatin-derived synthetic peptide shows antitumor activity in vivo. Bioconjug Chem. 2008;19:1980-6.

3. Hong $S$, Haibing $H$, Jialiang $H$, Xiaojuan $Z$, Jingjing $W$, Wenjing $W$, et al. PEGylated HM-3 presents anti-rheumatic bioactivity by inhibiting angiogenesis and inflammation. J Mater Chem B. 2014;2:800-13.

4. Liu Z, Wang F, Chen X. Integrin a(v) $\beta(3)$-Targeted Cancer Therapy. Drug Dev Res. 2008:69:329-39.

5. Xu H, Pan L, Ren Y, Yang Y, Huang X, Liu Z. RGD-modified angiogenesis inhibitor HM-3 dose: dual function during cancer treatment. Bioconjug Chem. 2011;22:1386-93.

6. Hodivala-Dilke K. alphavbeta3 integrin and angiogenesis: a moody integrin in a changing environment. Curr Opin Cell Biol. 2008:20:514-9.

7. Jain RK. Antiangiogenesis strategies revisited: from starving tumors to alleviating hypoxia. Cancer Cell. 2014;26:605-22.

8. Harrison H, Rogerson L, Gregson HJ, Brennan KR, Clarke RB, Landberg G. Contrasting hypoxic effects on breast cancer stem cell hierarchy is dependent on ER-alpha status. Cancer Res. 2013;73:1420-33.

9. Zeng W, Liu P, Pan W, Singh SR, Wei Y. Hypoxia and hypoxia inducible factors in tumor metabolism. Cancer Lett. 2015;356:263-7.
10. Nakatsugawa M, Takahashi A, Hirohashi Y, Torigoe T, Inoda S, Murase M, et al. SOX2 is overexpressed in stem-like cells of human lung adenocarcinoma and augments the tumorigenicity. Lab Invest. 2011;91:1796-804.

11. Justilien V, Walsh MP, Ali SA, Thompson EA, Murray NR, Fields AP. The PRKCI and SOX2 oncogenes are coamplified and cooperate to activate Hedgehog signaling in lung squamous cell carcinoma. Cancer Cell. 2014;25:139-51.

12. Zhao C, Setrerrahmane S, Xu H. Enrichment and characterization of cancer stem cells from a human non-small cell lung cancer cell line. Oncol Rep. 2015:34:2126-32.

13. Xiang R, Liao D, Cheng T, Zhou H, Shi Q, Chuang TS, et al. Downregulation of transcription factor SOX2 in cancer stem cells suppresses growth and metastasis of lung cancer. Br J Cancer. 2011;104:1410-7.

14. Fang WT, Fan CC, Li SM, Jang TH, Lin HP, Shih NY, et al. Downregulation of a putative tumor suppressor BMP4 by SOX2 promotes growth of lung squamous cell carcinoma. Int J Cancer. 2014:135:809-19.

15. Blache CA, Manuel ER, Kaltcheva TI, Wong AN, Ellenhorn JD, Blazar BR, et al. Systemic delivery of Salmonella typhimurium transformed with IDO shRNA enhances intratumoral vector colonization and suppresses tumor growth. Cancer Res. 2012;72:6447-56.

16. Jiang Z, Zhao P, Zhou Z, Liu J, Qin L, Wang H. Using attenuated Salmonella typhi as tumor targeting vector for MDR1 siRNA delivery. Cancer Biol Ther. 2007:6:555-60.

17. Jiang T, Zhou C, Gu J, Liu Y, Zhao L, Li W, et al. Enhanced therapeutic effect of cisplatin on the prostate cancer in tumor-bearing mice by transfecting the attenuated Salmonella carrying a plasmid co-expressing p53 gene and mdm2 siRNA. Cancer Lett. 2013:337:133-42.

18. Herreros-Villanueva M, Zhang JS, Koenig A, Abel EV, Smyrk TC, Bamlet WR, et al. SOX2 promotes dedifferentiation and imparts stem cell-like features to pancreatic cancer cells. Oncogenesis. 2013;2, e61.

19. Ganai S, Arenas RB, Forbes NS. Tumour-targeted delivery of TRAIL using Salmonella typhimurium enhances breast cancer survival in mice. $\mathrm{Br} J$ Cancer. 2009:101:1683-91.

20. Clairmont C, Lee KC, Ittensohn M. Biodistribution and Genetic Stability of the Novel Antitumor Agent VNP20009, a Genetically Modified Strain of Salmonella typhimurium. JID. 2000;181:1996-2002.

21. Chou Y-T, Lee C-C, Hsiao S-H, Lin S-E, Lin S-C, Chung C-H, et al. The Emerging Role of SOX2 in Cell Proliferation and Survival and Its Crosstalk with Oncogenic Signaling in Lung Cancer. Stem Cells. 2013:31:2607-19.

22. Ganai S, Arenas RB, Sauer JP, Bentley B, Forbes NS. In tumors Salmonella migrate away from vasculature toward the transition zone and induce apoptosis. Cancer Gene Ther. 2011;18:457-66.

23. Luo X, Li Z, Lin S, Le T, Ittensohn M, Bermudes D, et al. Antitumor effect of VNP20009, an attenuated Salmonella, in murine tumor models. Oncol Res. 2001;12:501-8.

24. Lippolis C, Refolo MG, D'Alessandro R, Carella N, Messa C, Cavallini A, et al. Resistance to multikinase inhibitor actions mediated by insulin like growth factor-1. J Exp Clin Cancer Res. 2015:34:90.

25. Qin Q, Furong W, Baosheng L. Multiple functions of hypoxia-regulated miR210 in cancer. J Exp Clin Cancer Res. 2014:33:50.

26. Murakami A, Takahashi F, Nurwidya F, Kobayashi I, Minakata K, Hashimoto $M$, et al. Hypoxia increases gefitinib-resistant lung cancer stem cells through the activation of insulin-like growth factor 1 receptor. PLoS One. 2014;9, e86459.

27. Bottsford-Miller JN, Coleman RL, Sood AK. Resistance and escape from antiangiogenesis therapy: clinical implications and future strategies. J Clin Oncol. 2012;30:4026-34

28. Niero EL, Rocha-Sales B, Lauand C, Cortez BA, de Souza MM, RezendeTeixeira $P$, et al. The multiple facets of drug resistance: one history, different approaches. J Exp Clin Cancer Res. 2014;33:37.

29. Yu B, Yang M, Shi L, Yao Y, Jiang Q, Li X, et al. Explicit hypoxia targeting with tumor suppression by creating an "obligate" anaerobic Salmonella Typhimurium strain. Sci Rep. 2012;2:436.

30. Sholl LM, Barletta JA, Yeap BY, Chirieac LR, Hornick JL. Sox2 protein expression is an independent poor prognostic indicator in stage I lung adenocarcinoma. Am J Surg Pathol. 2010;34:1193-8.

31. Piva M, Domenici G, Iriondo O, Rabano M, Simoes BM, Comaills V, et al. Sox2 promotes tamoxifen resistance in breast cancer cells. EMBO Mol Med. 2014;6:66-79.

32. Chou MY, Hu FW, Yu CH, Yu CC. Sox2 expression involvement in the oncogenicity and radiochemoresistance of oral cancer stem cells. Oral Oncol. 2015;51:31-9. 
33. Logue JS, Morrison DK. Complexity in the signaling network: insights from the use of targeted inhibitors in cancer therapy. Genes Dev. 2012;26:641-50.

34. Hanahan D, Weinberg RA. Hallmarks of cancer: the next generation. Cell. 2011:144:646-74.

35. Matuskova M, Kozovska Z, Toro L, Durinikova E, Tyciakova S, Cierna Z, et al. Combined enzyme/prodrug treatment by genetically engineered AT-MSC exerts synergy and inhibits growth of MDA-MB-231 induced lung metastases. J Exp Clin Cancer Res. 2015;34:33.

36. Yue D, Li H, Che J, Zhang Y, Tseng HH, Jin JQ, et al. Hedgehog/Gli promotes epithelial-mesenchymal transition in lung squamous cell carcinomas. J Exp Clin Cancer Res. 2014;33:34.

Submit your next manuscript to BioMed Central and we will help you at every step:

- We accept pre-submission inquiries

- Our selector tool helps you to find the most relevant journal

- We provide round the clock customer support

- Convenient online submission

- Thorough peer review

- Inclusion in PubMed and all major indexing services

- Maximum visibility for your research

Submit your manuscript at www.biomedcentral.com/submit
Biomed Central 